\title{
Long non-coding RNA ASncmtRNA-2 is upregulated in diabetic kidneys and high glucose-treated mesangial cells
}

\author{
YAN GAO ${ }^{1}$, ZHAO-YU CHEN ${ }^{2}$, YAN WANG $^{3}$, YAN LIU $^{3}$, JIAN-XIA MA $^{3}$ and YU-KUN LI ${ }^{3}$ \\ ${ }^{1}$ Department of Endocrinology, The Second Hospital of Hebei Medical University, Shijiazhuang, Hebei 050000; \\ ${ }^{2}$ Arthritis Clinic and Research Center, Peking University People's Hospital, Beijing 100044; \\ ${ }^{3}$ Department of Endocrinology, Third Hospital of Hebei Medical University, Shijiazhuang, Hebei 050051, P.R. China
}

Received September 12, 2015; Accepted November 4, 2016

DOI: $10.3892 /$ etm.2017.4027

\begin{abstract}
Diabetic nephropathy (DN) is one of the most frequent complications associated with type I and II diabetes mellitus. Kidneys from patients with DN are characterized by mesangial matrix expansion and increased thickness of the glomerular basement membrane, which are induced by reactive oxygen species (ROS) production. Previous studies have been conducted to investigate this; however, the detailed mechanism of DN progression remains to be elucidated. The present study evaluated the expression of antisense mitochondrial non-coding RNA-2 (ASncmtRNA-2) in an experimental DN model and cultured human mesangial cells. When mice that exhibited genetic type II diabetes developed DN, ASncmtRNA-2 expression was significantly increased $(\mathrm{P}=0.017)$ and was positively correlated with pro-fibrotic factor transforming growth factor $\beta 1$ (TGF $\beta 1$ ) expression and its downstream gene, fibronectin. Inhibition of ROS through administration of the nitric oxide synthase inhibitor, NG-nitro-L-Arginine methylester (L-NAME), significantly reduced $(\mathrm{P}=0.022)$ the upregulation of ASncmtRNA-2 in DN. In cultured human renal mesangial cells (HRMCs), ASncmtRNA-2 was upregulated by high glucose stimuli in a time-dependent manner. Glucose-induced upregulation of ASncmtRNA-2 was also reduced by co-incubation of HRMCs with L-NAME. Notably, specific short hairpin RNA against ASncmtRNA-2 significantly downregulated the expression of TGF $\beta 1$ in HRMCs. The present study suggests that ASncmtRNA-2 is upregulated by ROS and may promote glomerular fibrosis in DN via positively regulating the expression of pro-fibrotic factors. These findings may provide novel potential therapeutic and preventative treatments for DN.
\end{abstract}

Correspondence to: Dr Yu-Kun Li, Department of Endocrinology, Third Hospital of Hebei Medical University, 215 Heping Xi Road, Shijiazhuang, Hebei 050051, P.R. China

E-mail: yukunli845@gmail.com

Key words: antisense mitochondrial noncoding RNA2, diabetic nephropathy, oxidative stress, NL-nitro-L-arginine methyl ester, high glucose

\section{Introduction}

Diabetes mellitus (DM) is one of the most prevalent metabolic disorders worldwide, particularly in western countries. Over the past few decades, incidence rates of DM have markedly increased due to unhealthy diet, lack of exercise and a deteriorating environment $(1,2)$. DM may lead to complications such as diabetic nephropathy (DN), which is the leading cause of end stage renal disease in the USA (3). Diabetic kidneys are characterized by the development of albuminuria, enhanced glomerular filtration and pressure inside the glomerulus $(4,5)$. Of all patients with type I and II DM, 1/3 are affected by $\mathrm{DN}$ in their daily life (4). As DN progresses, excessive reactive oxygen species (ROS) are generated, inducing irreversible renal damage due to continuous hyperglycemic conditions $(6,7)$. Various enzymatic signaling pathways that generate ROS, such as nitric oxide synthase (NOS) and xanthine oxidase (XO) are stimulated in DN (8). However, the specific mechanism by which ROS regulates DN remains unclear.

A large proportion of regulatory RNAs are long non-coding RNAs (lncRNAs), which typically contain $>200$ nucleotides and serve important roles in the cytoplasm and nucleus (9). LncRNAs have been studied extensively in recent years, leading to the discovery of their important roles in tumorigenesis $(9,10)$, RNA transcription (11) and mRNA translation $(12,13)$. Furthermore, it has been demonstrated that IncRNAs are associated with DM progression (14). For example, IncRNA H19 is reportedly associated with the gestational DM-induced transformation of islet structure and function (14) and expression of the colorectal neoplasia differentially expressed lncRNA is affected by insulin and insulin-like growth factor signaling pathways in DM $(15,16)$.

Although it has recently been revealed that lncRNAs are associated with the progression of DM (17), no lncRNA of mitochondrial origin has been identified that is associated with DM or its complications. It is well-documented that mitochondria maintain ROS levels; high levels of ROS induce dysfunction of mitochondria, which in turn produce ROS at a higher rate (18). Due to the central roles of mitochondria in ROS production and ROS-mediated oxidative stress, it was hypothesized that lncRNAs transcribed from the mitochondrial genome may serve important roles in oxidative stress-mediated disease progression, such as DN. 
Antisense mitochondrial non-coding RNA-2 (ASncmtRNA-2; 2,104 nt) is a mitochondrial lncRNA that is expressed in the mitochondria and exported to the nucleus (19-21). Previous studies have revealed that it is involved in the tumorigenesis and mitochondrial retrograde signaling pathways (19-21). Notably, it has recently been demonstrated that ASncmtRNA-2 is overexpressed during aging and replicative senescence in human endothelial cells (18). This novel study indicated that ASncmtRNA-2 potentially serves a role in physiological oxidative stress. However, it remains unclear whether ASncmtRNA-2 is associated with pathological oxidative stress, such as the conditions observed in DN.

The present study aimed to investigate the role of ASncmtRNA-2 in oxidative stress-mediated DN fibrosis. Expression of ASncmtRNA-2 under ROS stimuli was assessed in an experimental model of DN and in cultured mesangial cells, and the possible mechanism through which ASncmtRNA-2 functions in DN was investigated.

\section{Materials and methods}

Experimental DN mouse model. A total of 16 four-week-old male C57BLKS/J mice weighing 15-18 g were purchased from the Model Animal Research Center of Nanjing University (Nanjing, China) and used as a control group. A total of 32 four-week old male C57BLKS/J-Lepr ${ }^{-}(\mathrm{db} / \mathrm{db})$ mice (weights, 30-32 g, Model Animal Research Center of Nanjing University), which genetically develop DM shortly following birth, were randomly divided into two groups: A DN group and a DN+NG-nitro-L-Arginine methylester (L-NAME) group ( $\mathrm{n}=16$ each). L-NAME, which is an NOS inhibitor that also inhibits ROS, was purchased from Santa Cruz Biotechnology, Inc. (Dallas, TX, USA). All mice were housed at $22^{\circ} \mathrm{C}, 45 \%$ humidity and a 12-h light/dark cycle. Mice had ad libitum access to water and food. The drinking water supply for the mice from the DN+L-NAME group contained L-NAME $(90 \mathrm{mg} / \mathrm{kg}$ ) throughout the experiment (22). In each group, RNA from the kidneys was harvested at weeks eight and 16 from three randomly selected mice. Remaining mice were sacrificed with $1.5 \%$ Pelltobarbitalum Natricum $(0.1 \mathrm{ml} / 20 \mathrm{~g}$; Sigma-Aldrich; Merck KGaA, Darmstadt, Germany) at week 24 and blood was harvested for biochemical analysis. Urine samples were collected at the same time and the urine albumin to creatinine ratio (UACR) was assessed. The present study was approved by the Institutional Animal Care and Use Committee of Hebei Medical University (Shijiazhuang, China).

Hematoxylin and eosin, periodic acid-Schiff and trichrome Masson staining. Tissue samples were fixed in $10 \%$ buffered formalin, embedded in paraffin and spliced into $5-\mu \mathrm{m}$ sections. Afterwards, hematoxylin and eosin staining, periodic acid-Schiff staining, as well as Trichrome Masson staining were performed as described before (23).

Cell culture. Human mesangial cells (HRMCs) were purchased from ATCC (Manassas, VA, USA) and cultured in RPMI-1640 medium (Gibco; Thermo Fisher Scientific, Inc., Waltham, MA, USA) supplemented with $10 \%$ fetal bovine serum (Gibco; Thermo Fisher Scientific, Inc.). Cells were seeded in a six-well plate and transfected with specific short hairpin RNA (shRNA) to silence ASncmtRNA-2 (Sigma-Aldrich) using Lipofectamine 2000 (Invitrogen; Thermo Fisher Scientific, Inc.). Culture medium was replenished $6 \mathrm{~h}$ post-transfection. Cells were incubated at $37^{\circ} \mathrm{C}$ for $24 \mathrm{~h}$ and harvested for subsequent analysis. Due to the lack of commercial primary antibody against ASncmtRNA-2, only mRNA levels were detected throughout the study.

$R N A$ isolation and reverse transcription-quantitative polymerase chain reaction ( $R T-q P C R)$. RNA from mouse tissues and cultured cells were isolated using TRIzol reagent (Takara Biotechnology Co., Ltd., Dalian, China) and incubated at $37^{\circ} \mathrm{C}$ with DNase I (Invitrogen; Thermo Fisher Scientific, Inc.) according to the manufacturers' protocols. A total of $1 \mu \mathrm{g}$ RNA was reverse transcribed into cDNA with a volume of $20 \mu \mathrm{l}$ (Takara Biotechnology Co., Ltd.) and qPCR was subsequently performed using an ABI PRISM 7900 system with a SYBR Premix Ex Taq Perfect Real-Time kit (Takara Biotechnology Co., Ltd.). Briefly, PCR was performed as follows: Initial denaturation at $95^{\circ} \mathrm{C}$ for $2 \mathrm{~min}, 35$ cycles at $95^{\circ} \mathrm{C}$ for $30 \mathrm{sec}$ and at $55^{\circ} \mathrm{C}$ for $60 \mathrm{sec}$. Fluorescence was recorded following each cycle using the ABI7900 (Applied Biosystems; Thermo Fisher Scientific, Inc.). GAPDH was included as an internal reference gene. Primers for corresponding genes are listed in Table I. Each assay was performed in triplicate unless otherwise stated. The gene expression was normalized to GAPDH with the delta delta $\mathrm{Ct}$ method as explained in details in previous article (24).

Measurement of intracellular ROS. To detect the effects of L-NAME and high glucose on ROS production, the intracellular ROS level was evaluatedusinganon-fluorescent probe, 2',7'-dichlorofluorescein diacetate (DCFH-DA; Beyotime Institute of Biotechnology, Nanjing, China). Briefly, HRMCs were seeded in a 96 -well plate $\left(1 \times 10^{5}\right.$ cells/well) and cultured with low (LG) or high glucose (HG), with each medium supplemented with or without L-NAME. Culture medium was removed and plates were washed with PBS three times at $24 \mathrm{~h}$ following culture. DCFH-DA was diluted with DCFH/F12 to a final concentration of $10 \mu \mathrm{M}$ and co-incubated with HRMCs at $37^{\circ} \mathrm{C}$ for $20 \mathrm{~min}$. Fluorescence was measured using a Tecan fluorescence plate reader (Tecan Group, Ltd., Männedorf, Switzerland) at $485 \mathrm{~nm}$ for excitation and $530 \mathrm{~nm}$ for emission. Each measurement was performed in triplicate and repeated at least three times.

Statistical analysis. Data were presented as the mean \pm standard deviation and analyzed using a Student's t-test. Statistical analyses were performed using Excel software (Microsoft, Inc., Richmond, WA, USA). Results were obtained from three independent experiments and $\mathrm{P}<0.05$ was considered to indicate a statistically significant difference.

\section{Results}

Establishment of experimental DN model. To assess the expression of ASncmtRNA-2 in DN samples and ROS-reduced diabetic kidney samples, a mouse model of DN was established. L-NAME is anNOS inhibitor that is able to reduce the production of ROS in diabetic kidneys (25), hence administering 
Table I. Primers used in quantitative polymerase chain reaction.

Genes

hASncmtRNA-2

hTGF $\beta 1$

$\mathrm{hFN}$

hGAPDH

mASncmtRNA-2

mTGF $\beta 1$

$\mathrm{mFN}$

mGAPDH
Primer sequence

Forward: 5'-ACCGTGCAAAGGTAGCATAATCACT-3'

Reverse: 5'-CCGTAAATGATATCATCTCAACT-3'

Forward: 5'-TGCACATTGCCTGTTCTGCT-3'

Reverse: 5'-TGCATCTTGGTTGGCTGCAT-3'

Forward: 5'-TGCACATTGCCTGTTCTGCT-3'

Reverse: 5'-TGCATCTTGGTTGGCTGCAT-3'

Forward: 5'-CTGACTTCAACAGCGACACC-3'

Reverse: 5'-TGCTGTAGCCAAATTCGTTGT-3'

Forward: 5'-GCTAAACGAGGGTCCAACTG-3'

Reverse: 5'-CTAACCTAGAGAAGGTTATTAGGTTT-3'

Forward: 5'-TAAAGAGGTCACCCGCGTGCTAAT-3'

Reverse: 5'-ACTGCTTCCCGAATGTCTGACGTA-3'

Forward: 5'-ACGGTTCCCATTACGCCAT-3'

Reverse: 5'-TCATCCGCTGGCCATTTTCT-3'

Forward: 5'-AGGTCGGTGTGAACGGATTTG-3'

Reverse: 5'-TGTAGACCATGTAGTTGAGGTCA-3'

h, human; m, mouse; ASncmtRNA-2, antisense mitochondrial non-coding RNA-2; TGF $\beta 1$, transforming growth factor $\beta 1$; FN, fibronectin.

Table II. Physical and biochemical parameters after 24 weeks of study in control, DN and DN+L-NAME group mice.

\begin{tabular}{lrrr}
\hline Parameters & Control & DN & DN + L-NAME \\
\hline Number & \multicolumn{1}{c}{10} & $9^{\mathrm{a}}$ & 10 \\
Body weight (g) & $22.09 \pm 2.05$ & $41.93 \pm 1.32$ & $47.97 \pm 0.87$ \\
Kidney/body weight ratio (\%) & $0.45 \pm 0.12$ & $0.39 \pm 0.28$ & $0.31 \pm 0.31$ \\
Glucose (mmol/l) & $6.72 \pm 0.01$ & $23.80 \pm 0.01$ & $14.41 \pm 0.009$ \\
HbAlc (\%) & $5.3 \pm 0.09$ & $9.8 \pm 0.34$ & $7.6 \pm 0.12$ \\
HbAlc (mmol/mol) & $34.426 \pm 0.98$ & $83.607 \pm 3.76$ & $59.563 \pm 1.31$ \\
Total cholesterol (mmol/l) & $4.9 \pm 0.17$ & $11.8 \pm 1.01$ & $9.4 \pm 0.97$ \\
Triglycerides (mmol/l) & $1.5 \pm 0.24$ & $3.7 \pm 0.33$ & $2.6 \pm 0.42$ \\
HDL (mmol/l) & $1.3 \pm 0.32$ & $2.7 \pm 0.11$ & $1.7 \pm 0.21$ \\
LDL (mmol/l) & $4.5 \pm 0.56$ & $10.3 \pm 0.31$ & $7.4 \pm 0.11$ \\
\hline
\end{tabular}

Data are presented as the mean \pm standard deviation. ${ }^{a}$ One mouse from this group succumbed at the 22nd week. DN, diabetic nephropathy; L-NAME, NG-nitro-L-Arginine methylester; HbA1c, glycated hemoglobin; HDL, high density lipoprotein; LDL, low density lipoprotein.

diabetic mice with L-NAME is an effective method to evaluate the effects of reduced ROS on the expression of ASncmtRNA-2 in diabetic kidneys. At week 24, blood samples were harvested from each group to analyze physical and biochemical parameters (Table II), including glucose, glycated hemoglobin, total cholesterol, triglycerides, high-density lipoproteins and low-density lipoproteins. The differences observed in these parameters between groups demonstrated that mice from the DN group were suffering from a metabolic disorder whereas those in the control group were not and that administration of L-NAME was able to relieve these disorders. These findings suggest that mice with genetically developed DM developed DN at week 24, and L-NAME effectively ameliorated
ROS-mediated renal damage in the experimental DN model. Mice were sacrificed by the end of week 24 and their kidneys were harvested. Half of each kidney was formalin-fixed and paraffin-embedded for histological analysis whereas the other half was frozen to allow for isolation of tissue RNA.

Urine samples were collected at weeks 8, 16 and 24 to assess renal function. As illustrated in Fig. 1A, there was no significant difference in UACR over time for control mice. A significant progressive increase in UACR was observed in DN mice over time compared with the control group $(\mathrm{P}<0.05)$. In the DN+L-NAME group, UACR was also significantly increased compared with the control group $(\mathrm{P}<0.05)$; however, the UCAR levels in this group were markedly lower than 
A

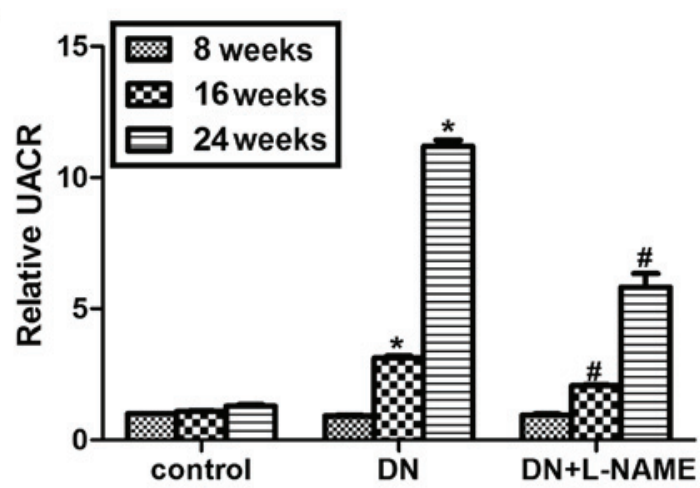

B

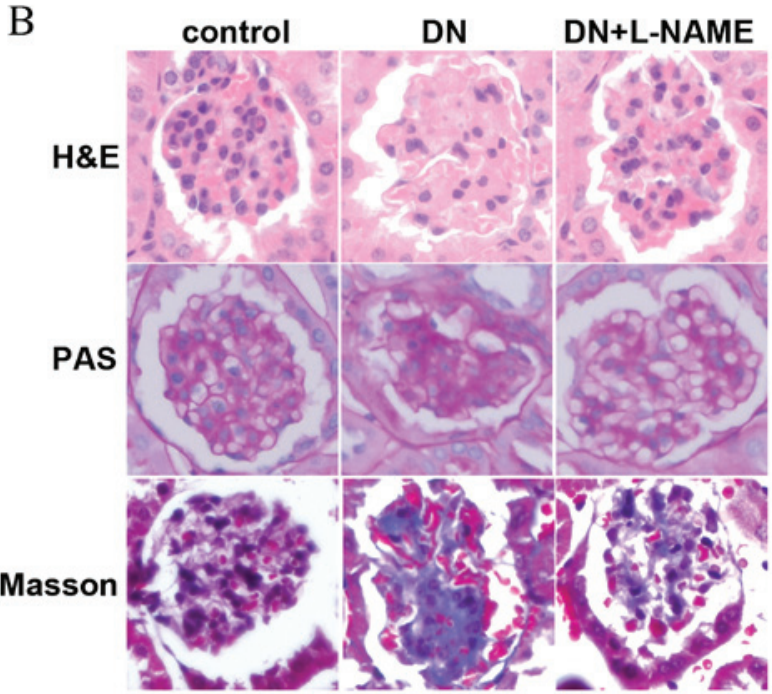

Figure 1. Establishment of an experimental mouse model of DN. (A) Relative UACR in each group at different time intervals (weeks 8, 16 and 24). (B) H\&E, PAS and Trichrome Masson staining were performed to examine the pathological changes in dissected kidneys from each group. Mice were fed for 24 weeks and experienced significant renal damage as evidenced by the UACR and other parameters presented in Table II. "P<0.05, DN vs. control; ${ }^{\text {"}} \mathrm{P}<0.05, \mathrm{DN}+\mathrm{L}-\mathrm{NAME}$ vs. control. $\mathrm{DN}$, diabetic nephropathy; UACR, urine albumin $(\mu \mathrm{g})$ to creatinine $(\mu \mathrm{g})$ ratio; H\&E, haemotoxylin and eosin; PAS, Periodic acid-Schiff; L-NAME, NG-nitro-L-Arginine methylester.

the paired mice from the DN group, irrespective of time. As demonstrated in Fig. 1B, mice from the DN group exhibited more severe glomerular lesions as compared with control mice. In particular, the cellular architecture in the glomeruli was less revealed and the extracellular matrix architecture was notably damaged in this group; furthermore, increased matrix deposition, glycogen accumulation and collagen production were observed via hematoxylin and eosin, periodic acid-Schiff and trichrome Masson staining, respectively. By contrast, tissue from mice with DN that were administered L-NAME exhibited reduced renal damage compared with the DN group, with less damaged cell architecture and matrix deposition. These data indicate the successful establishment of an experimental DN model from a genetically DM-developed mouse strain, and suggest that administering the L-NAME ROS-inhibitor effectively ameliorated renal damage.

Expression of ASncmtRNA-2 is upregulated in mice with DN and decreased by $L-N A M E$. Three mice from each group were randomly chosen and sacrificed at weeks 8, 16 and 24 to assess
A

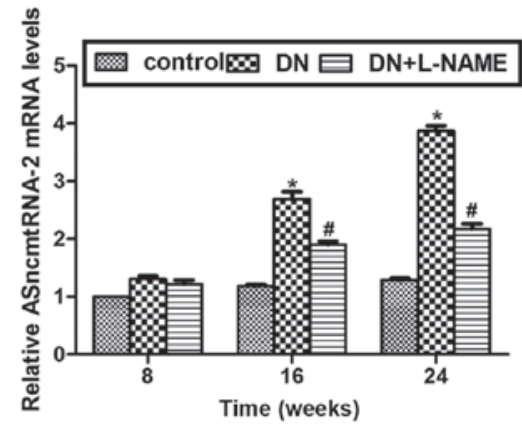

B

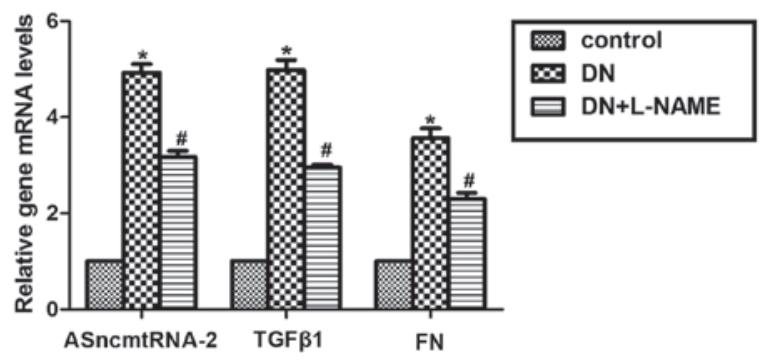

C

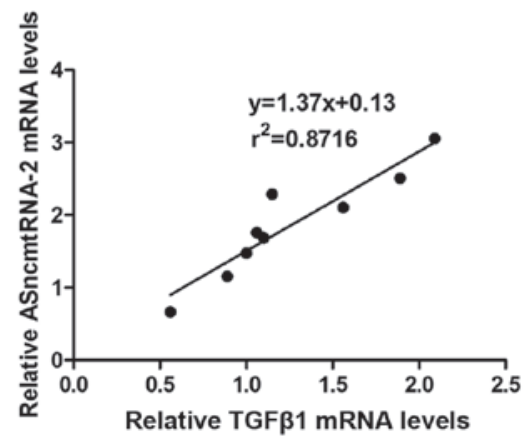

Figure 2. Expression of ASncmtRNA-2 is upregulated in DN and reduced by L-NAME. (A) RT-qPCR analysis revealed the expression of ASncmtRNA-2 in the mice model at weeks 8, 16 and 24. Expression of ASncmtRNA-2 was increased in mice with DN but reduced by L-NAME in a time-dependent manner. (B) Mice in each group were sacrificed at 24 weeks, and total RNA was isolated and assessed via RTq-PCR analysis to measure the mRNA levels of ASncmtRNA-2, TGF $\beta 1$ and FN. mRNA expression levels of ASncmtRNA-2 were increased in DN samples, but were subsequently decreased by L-NAME administration. mRNA levels of TGF $\beta 1$ and FN displayed similar expression patterns. (C) Linear regression analysis suggests the mRNA levels of ASncmtRNA-2 are positively correlated with that of TGF $\beta 1$ in the experimental model of DN. $n=9$ (one mouse died prior to week 24). $\mathrm{r}^{2}=0.8716 .{ }^{*} \mathrm{P}<0.05, \mathrm{DN}$ vs. control; ${ }^{~} \mathrm{P}<0.05, \mathrm{DN}+\mathrm{L}-\mathrm{NAME}$ vs. control. ASncmtRNA-2, antisense mitochondrial non-coding RNA-2; DN, diabetic nephropathy; L-NAME, NG-nitro-L-Arginine methylester; RT-qPCR, reverse transcription-quantitative polymerase chain reaction; TGF, transforming growth factor; FN, fibronectin.

the expression levels of ASncmtRNA-2. Data from RT-qPCR revealed that the mRNA expression level of ASncmtRNA-2 in the DN group was significantly increased (2.6-fold) compared with control mice at week 16, and 3.8-fold at week 24 (both $\mathrm{P}<0.05)$. Furthermore, the mRNA expression levels of ASncmtRNA-2 in the DN+L-NAME group were significantly higher $(\mathrm{P}<0.05)$ than the control group and markedly lower than the DN group at weeks 16 and 24 (Fig. 2A). These results indicate that ASncmtRNA-2 increased throughout the development of DN and that inhibition of ROS by L-NAME may reduce the upregulation of ASncmtRNA-2 in DN. Transforming growth factor $\beta 1$ (TGF $\beta 1$ ) and its downstream gene, fibronectin (FN), are well-known pro-fibrotic factors in DN (26). To investigate 
A

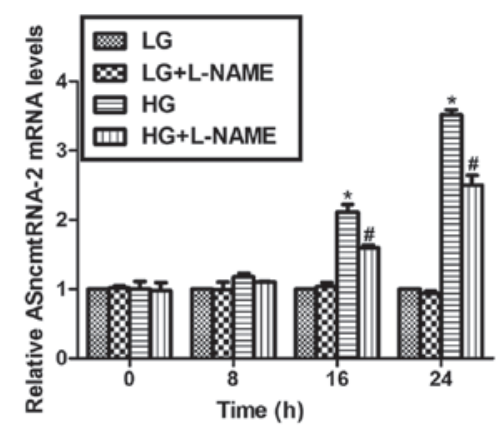

B
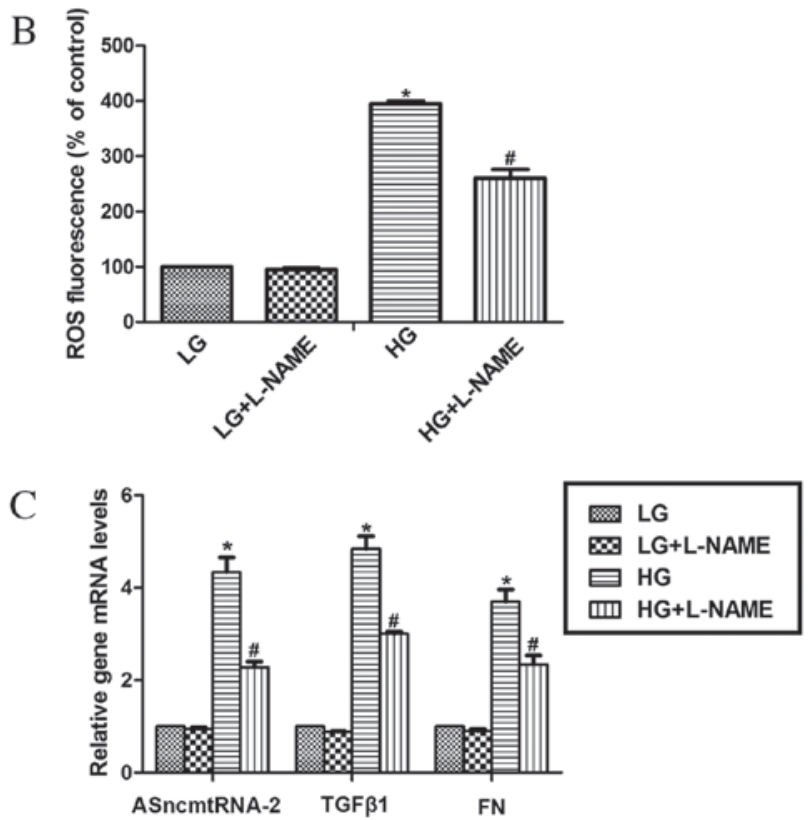

Figure 3. ASncmtRNA-2 is upregulated in HG-treated HRMCs. (A) HRMCs with the indicated treatments were harvested at $0,8,16$ or $24 \mathrm{~h}$. mRNA levels of ASncmtRNA-2 at each time point were recorded. (B) Relative ROS fluorescence levels were detected in HG and HRMCs with or without L-NAME treatment using dichloro-dihydro-fluorescein diacetate probes. (C) mRNA levels of TGF $\beta 1$ and FN were analyzed via RT-qPCR assay at $24 \mathrm{~h}$ post-treatment. ${ }^{*} \mathrm{P}<0.05$ vs. $\mathrm{LG} ;{ }^{*} \mathrm{P}<0.05$ vs. $\mathrm{LG}$. ASncmtRNA-2, antisense mitochondrial non-coding RNA-2; ROS, reactive oxygen species; HG, high glucose; LG, low glucose; L-NAME, NG-nitro-L-Arginine methylester; HRMC, human renal mesangial cell; TGF $\beta 1$, transforming growth factor $\beta 1$ FN, fibronectin; RT-PCR, revere transcription polymerase chain reaction.

whether the expression of ASncmtRNA-2 is associated with these pro-fibrotic factors, mRNA levels of TGF $\beta 1$ and FN were detected in mouse kidneys using RT-qPCR analysis. As demonstrated in Fig. 2B, the mRNA levels of TGF $\beta 1$ and FN were significantly upregulated in the DN group compared with the control group, $(\mathrm{P}<0.05)$ which were markedly decreased following treatment with L-NAME (DN vs. DN+L-NAME). Compared with the control group, mRNA levels of TGF $\beta 1$ and FN were significantly upregulated in the DN+L-NAME group $(\mathrm{P}<0.05)$. These findings suggest that there may be an association between TGF $\beta 1$ and ASncmtRNA-2 levels. The expression of ASncmtRNA-2 was compared with that of TGF $\beta 1$ in mice with DN sacrificed at week $24(n=9)$, which indicated that they were positively correlated, with a coefficient of determination $\left(\mathrm{r}^{2}\right)$ as 0.8716 (Fig. 2C). These results suggest that ASncmtRNA-2 is significantly overexpressed in DN and positively correlated with expression of pro-fibrotic factor TGF $\beta 1$.

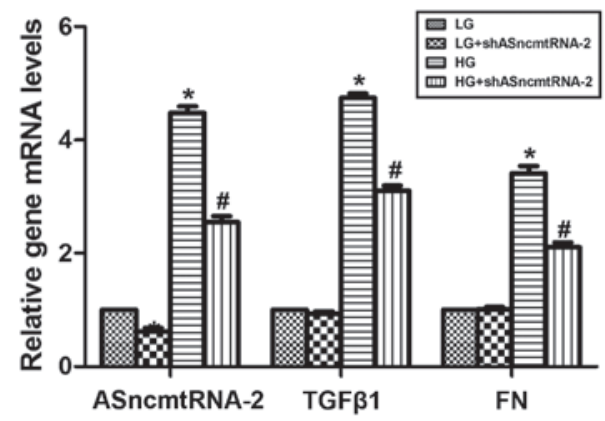

Figure 4. Knockdown of ASncmtRNA-2 inhibits the expression of TGF $\beta 1$ in HG-treated HRMCs. Specific ASncmtRNA-2-silencing shRNA were transfected into HRMCs in vitro and a scramble shRNA was transfected for control. Cells were cultured in LG or HG conditions with or without the specific shRNA. mRNA levelsof ASncmtRNA-2, TGF $\beta 1$ and FN were determined via reverse transcription-quantitative polymerase chain reaction. ${ }^{*} \mathrm{P}<0.05$, HG vs. LG; ${ }^{\mathrm{P}}<0.05, \mathrm{HG}+\mathrm{shASncmtRNA}-2$ vs. HG. ASncmtRNA-2, antisense mitochondrial non-coding RNA-2; TGF $\beta 1$, transforming growth factor $\beta 1$; HRMC, human renal mesangial cell; LG, low glucose; HG, high glucose; shRNA, short hairpin RNA; FN, fibronectin.

ASncmtRNA-2 is upregluated in cultured HRMCs treated with $H G$. Based on the experimental observations thus far, the expression levels of ASncmtRNA-2 in cultured HRMCs were studied. Cells were seeded into six-well plates and cultured with LG or HGmedium, with or without additional L-NAME treatment. mRNA levels of ASncmtRNA-2 were measured at different time intervals. At $8 \mathrm{~h}$ post-treatment, expression of ASncmtRNA-2 remained unchanged in all groups, as compared with $0 \mathrm{~h}$. However, the level of ASncmtRNA-2 mRNA increased significantly in HG and HG+L-NAME-treated cells compared with LG-treated cells from $16 \mathrm{~h}$ onwards $(\mathrm{P}<0.05)$. Although a significant increase was also observed in the ASncmtRNA-2mRNA level in the HG+L-NAME group vs. the control group, this was markedly reduced compared with the HG group (Fig. 3A). Relative ROS levels in HRMCs were detected with the non-fluorescent probe DCFH-DA. DCFH reacts with cellular ROS to generate the fluorescent product DCF, which can be easily traced within cells. Therefore, intracellular ROS levels were determined using a DCFH-DC probe as described previously (27). As shown in Fig. 3B, ROS fluorescence was significantly upregulated in HG-treated cells $(\mathrm{P}<0.05)$, but was suppressed by $37.5 \%$ when HG cells were co-incubated with L-NAME, suggesting that L-NAME effectively inhibits ROS production. Levels of ASncmtRNA-2mRNA, TGF $\beta 1$ and FN also displayed similar expression patterns (Fig. 3C). These data suggest that ASncmtRNA-2 is also overexpressed in mesangial cells exposed to $\mathrm{HG}$, which is the in vivo environment of patients with DM. Under HG stimuli, expression of ASncmtRNA-2 is positively associated with that of TGF $\beta 1$ in vitro.

Knockdown of ASncmtRNA-2 inhibits the expression of TGF $\beta 1$ in HG-treated HRMCs. The role of ASncmtRNA-2 in regulating the expression of TGF $\beta 1$ was evaluated using a specific ASncmtRNA-2-silencing shRNA to knockdown expression of ASncmtRNA-2. As indicated in Fig. 4, the levels of ASncmtRNA-2 mRNA decreased to $60-70 \%$ by specific silencing shRNA in LG- and HG-treated cells. Furthermore, the expression of ASncmtRNA-2, TGF $\beta 1$ and FN were 
significantly increased in HG conditions as compared with LG $(\mathrm{P}<0.05)$. ASncmtRNA-2 was significantly depleted by shASncmtRNA-2 in the cells and mRNA levels of TGF $\beta 1$ and FN were significantly decreased by 34.8 and $44.4 \%(\mathrm{P}<0.05)$, respectively. These findings suggest that knockdown of ASncmtRNA-2 is able to decrease the expression of TGF $\beta 1$, which in turn indicates that ASncmtRNA-2 positively regulates pro-fibrotic factors during DN progression.

\section{Discussion}

There are many complications associated with DM, including $\mathrm{DN}$, which may lead to renal failure and eventual death (28). Overproduction of oxidative products, such as ROS, induces oxidative damage to the cellular and extracellular structures of the kidneys, particularly in HRMCs (29). Therefore, the present study used HRMCs to evaluate the expression of ASncmtRNA-2 upon HG and L-NAME stimulation. ROS are able to induce injury to human kidneys through the following mechanisms: i) Inducing lipid peroxidation, protein crosslinking and the formation of DNA adducts, leading to tissue damage; ii) inducing direct damage to cellular DNA; and iii) activating multiple cellular signaling pathways, including the nuclear factor- $\mathrm{kB}$ and TGF $\beta 1$ signaling pathways. These mechanisms induce further generation of ROS, secretion of cytokines, synthesis and deposition of extracellular matrix components, which induce more severe damage to kidneys $(30,31)$. Therefore, it is necessary to identify a novel method to regulate ROS generation to reduce renal damage in $\mathrm{DN}$ in a clinical setting.

LncRNAs are typically defined as noncoding RNA $>200 \mathrm{nt}$ in length that have been revealed to have roles in various diseases, typically acting as scaffolds for multiple proteins and forming complexes to regulate protein expression $(12,13)$. LncRNA UCA1 has previously been shown to contribute to cardiomyocyte apoptosis by suppressing the expression of P27, whose overexpression in heart injury is associated with enhanced ROS production and increased cell apoptosis (31). However, it remains to be elucidated whether other lncRNAs serve a role in mechanisms of oxidative stress.

Recently, studies of mitochondrial lncRNAs and their role in oxidative stress-induced damages have been conducted $(9,18)$. It has been demonstrated that mitochondrial dysfunction is associated with high ROS levels and vice versa (18); therefore, mitochondrial lncRNAs are considered to be a viable target for the regulation of ROS production. A recent pilot study demonstrated that mitochondrial lncRNAASncmtRNA-2 was increased in aging and replicative senescence in human endothelial cells (18), suggesting that mitochondrial ASncmtRNA-2 may be associated with ROS generation. The present study further demonstrates that ASncmtRNA-2is overexpressed in DN in vivo. Inhibition of ROS production through administering L-NAME significantly ameliorated renal damage in mice and decreased the expression of ASncmtRNA-2, suggesting that ASncmtRNA-2 may be a substrate of ROS and may execute the ROS-mediated effects in DN. In the HG-cultured HRMCs, ROS was increased and the expression of ASncmtRNA-2 exhibited a similar pattern, further suggesting that ROS are able to induce the expression of ASncmtRNA-2. This hypothesis is further supported by the fact that inhibition of ROS via L-NAME administration decreased ASncmtRNA-2 expression. These in vivo and in vitro data suggest that ASncmtRNA-2 is a substrate of ROS that may have critical roles in $\mathrm{DN}$ progression.

The present study demonstrated that increased expression of ASncmtRNA-2 may be associated with higher extracellular matrix deposition in glomeruli, suggesting that ASncmtNA-2 may regulate the glomerular fibrosis process during DN. Therefore, a key pro-fibrotic factor, TGF $\beta 1$, and its downstream target FN, were evaluated. It was discovered that mice with DN exhibited severe glomerular fibrosis with significant increases in TGF $\beta 1$ and FN expression levels. mRNA expression levels of ASncmtRNA-2 were positively correlated with that of TGF $\beta 1$ in the DN samples, and the in vitro data also revealed that HG induced increases in ASncmtRNA-2 and TGF $\beta 1$ levels. Knockdown of ASncmtRNA-2 by specific shRNA induced significant decreases in mRNA levels of TGF $\beta 1$ and FN. These data suggest that TGF $\beta 1$ is regulated by ASncmtRNA-2 in DN, and ROS-induced expression of ASncmtRNA-2 may contribute to DN fibrosis via regulating the pro-fibrotic factor TGF $\beta 1$. However, the detailed mechanisms of how ASncmtRNA-2 regulates TGF $\beta 1$ remain to be elucidated. Bianchessi et al (18) recently suggested that ASncmtRNA-2 may function as a non-canonical precursor of microRNA (miRNA), hsa-miR-4485 and hsa-miR-1973, as they originate from a mitochondrial transcript. However, further research is required to elucidate the regulatory network between TGF $\beta 1$, ASncmtRNA-2 and miRNA in DN.

In conclusion, ASncmtRNA-2expression is upregulated in DN. ROS-induced expression of ASncmtRNA-2 may contribute to DN fibrosis through regulating TGF $\beta 1$; however, the mechanisms by which ASncmtRNA-2 regulates TGF $\beta 1$ remain to be elucidated. Therapeutic agents targeting ASncmtRNA-2 may prove to be effective for the prevention and treatment of DN.

\section{References}

1. Forbes JM and Cooper ME: Mechanisms of diabetic complications. Physiol Rev 93: 137-188, 2013

2. Guariguata L, Whiting DR, Hambleton I, Beagley J, Linnenkamp U and Shaw JE: Global estimates of diabetes prevalence for 2013 and projections for 2035. Diabetes Res Clin Pract 103: 137-149, 2014.

3. Collins AJ, Foley RN, Chavers B, Gilbertson D, Herzog C, Johansen K, Kasiske B, Kutner N, Liu J, St Peter W, et al: 'United States renal data system 2011 annual data report: Atlas of chronic kidney disease \& end-stage renal disease in the United States. Am J Kidney Dis 59 (1 Suppl 1): A7, e1-e420, 2012.

4. Mogensen CE: Diabetic renal disease in patients with type 2 diabetes mellitus: New strategies for prevention and treatment. Treat Endocrinol 1: 3-11, 2002.

5. Remuzzi G, Macia M and Ruggenenti P: Prevention and treatment of diabetic renal disease in type 2 diabetes: The BENEDICT study. J Am Soc Nephrol 17 (4 Suppl 2): S90-S97, 2006.

6. Fridlyand LE and Philipson LH: Oxidative reactive species in cell injury: Mechanisms in diabetes mellitus and therapeutic approaches. Ann N Y Acad Sci 1066: 136-151, 2005.

7. Maiwulanjiang M, Chen J, Xin G, Gong AG, Miernisha A, Du CY, Lau KM, Lee PS, Chen J, Dong TT, et al: The volatile oil of Nardostachyos Radix et Rhizoma inhibits the oxidative stress-induced cell injury via reactive oxygen species scavenging and Akt activation in H9c2 cardiomyocyte. J Ethnopharmacol 153: 491-498, 2014.

8. Di Marco ED, Jha JC, Sharma A, Wilkinson-Berka JL, Jandeleit-Dahm KA and de Haan JB: Are reactive oxygen species still the basis for diabetic complications? Clin Sci (Lond) 129: 199-216, 2015. 
9. Qiu MT, Hu JW, Yin R and Xu L: Long noncoding RNA: An emerging paradigm of cancer research. Tumour Biol 34: 613-620, 2013.

10. Zhang H, Chen Z, Wang X, Huang Z, He Z and Chen Y: Long non-coding RNA: A new player in cancer. J Hematol Oncol 6 : 37, 2013.

11. Gibb EA, Warren RL, Wilson GW, Brown SD, Robertson GA, Morin GB and Holt RA: Activation of an endogenous retrovirus-associated long non-coding RNA in human adenocarcinoma. Genome Med 7: 22, 2015

12. Amit-Avraham I, Pozner G, Eshar S, Fastman Y, Kolevzon N, Yavin E and Dzikowski R: Antisense long noncoding RNAs regulate var gene activation in the malaria parasite Plasmodium falciparum. Proc Natl Acad Sci USA 112: E982-E991, 2015.

13. Hung CL, Wang LY, Yu YL, Chen HW, Srivastava S, Petrovics $\mathrm{G}$ and Kung $\mathrm{HJ}$ : A long noncoding RNA connects c-Myc to tumor metabolism. Proc Natl Acad Sci USA 111: 18697-18702, 2014.

14. Ding GL, Wang FF, Shu J, Tian S, Jiang Y, Zhang D, Wang N, Luo Q, Zhang Y, Jin F, et al: Transgenerational glucose intolerance with Igf2/H19 epigenetic alterations in mouse islet induced by intrauterine hyperglycemia. Diabetes 61: 1133-1142, 2012.

15. Ellis BC, Graham LD and Molloy PL: CRNDE, a long non-coding RNA responsive to insulin/IGF signaling, regulates genes involved in central metabolism. Biochim Biophys Acta 1843: 372-386, 2014

16. Kornfeld JW and Brüning JC: Regulation of metabolism by long, non-coding RNAs. Front Genet 5: 57, 2014.

17. Motterle A, Gattesco S, Caille D, Meda P and Regazzi R: Involvement of long non-coding RNAs in beta cell failure at the onset of type 1 diabetes in NOD mice. Diabetologia 58: $1827-1835,2015$

18. Bianchessi V, Badi I, Bertolotti M, Nigro P, D'Alessandra Y, Capogrossi MC, Zanobini M, Pompilio G, Raucci A and Lauri A: The mitochondrial lncRNA ASncmtRNA-2 is induced in aging and replicative senescence in Endothelial Cells. J Mol Cell Cardiol 81: 62-70, 2015

19. Burzio VA, Villota C, Villegas J, Landerer E, Boccardo E, Villa LL, Martínez R, Lopez C, Gaete F, Toro V, et al: Expression of a family of noncoding mitochondrial RNAs distinguishes normal from cancer cells. Proc Natl Acad Sci USA 106: 9430-9434, 2009.

20. Mercer TR, Neph S, Dinger ME, Crawford J, Smith MA, Shearwood AM, Haugen E, Bracken CP, Rackham O, Stamatoyannopoulos JA, et al: The human mitochondrial transcriptome. Cell 146: 645-658, 2011.
21. Rackham O, Shearwood AM, Mercer TR, Davies SM, Mattick JS and Filipovska A: Long noncoding RNAs are generated from the mitochondrial genome and regulated by nuclear-encoded proteins. RNA 17: 2085-2093, 2011.

22. Ocsan RJ, Lai YN, Prabhu KV, Hambly BD and McLachlan CS Chronic NG-nitro-l-arginine methyl ester (L-NAME) administration in C57BL/6J mice induces a sustained decrease in c-kit positive cells during development of cardiac hypertrophy. J Physiol Pharmacol 64: 727-736, 2013.

23. Margaritescu C, Raica M, Simionescu C, Mogoantă L, Surpăţeanu M, Jaubert F and Bogdan F: Tumoral stroma of salivary pleomorphic adenoma-histopathological, histochemical and immunohistochemical study. Rom J Morphol Embryol 46: 211-223, 2005

24. Livak KJ and Schmittgen TD: Analysis of relative gene expression data using real-time quantitative PCR and the 2(-Delta Delta C(T)) Method. Methods 25: 402-408, 2001.

25. Pan X, Wang X, Wang X, Sun Z, Zhang X, Liang X, Li Z and Dou Z: Nitric oxide regulates blastocyst hatching in mice. Int J Clin Exp Med 8: 6994-7001, 2015.

26. Merline R, Lazaroski S, Babelova A, Tsalastra-Greul W, Pfeilschifter J, Schluter KD, Gunther A, Iozzo RV, Schaefer RM and Schaefer L: Decorin deficiency in diabetic mice: Aggravation of nephropathy due to overexpression of profibrotic factors, enhanced apoptosis and mononuclear cell infiltration. J Physiol Pharmacol 60 (Suppl 4): S5-S13, 2009.

27. Tian YY, An LJ, Jiang L, Duan YL, Chen J and Jiang B: Catalpol protects dopaminergic neurons from LPS-induced neurotoxicity in mesencephalic neuron-glia cultures. Life Sci 80: 193-199, 2006.

28. Gordin D, Wadén J, Forsblom C, Thorn LM, RosengårdBärlund M, Heikkilä O, Saraheimo M, Tolonen N, Hietala K, Soro-Paavonen A, et al: Arterial stiffness and vascular complications in patients with type 1 diabetes: The Finnish Diabetic Nephropathy (FinnDiane) Study. Ann Med 44: 196-204, 2012.

29. Chandel NS and Budinger GR: The cellular basis for diverse responses to oxygen. Free Radic Biol Med 42: 165-174, 2007.

30. Brownlee M: The pathobiology of diabetic complications: A unifying mechanism. Diabetes 54: 1615-1625, 2005.

31. Giacco F and Brownlee M: Oxidative stress and diabetic complications. Circ Res 107: 1058-1070, 2010. 